\title{
Managed Care Workshop Schweiz - Zukunftserfindung 2014
}

\author{
Am 16. Managed Care Workshop vom 12. bis 14. Dezember 2013 in Scuol trafen \\ sich erneut wichtige Player der Managed-Care-Szene zum jährlichen Gedankenaus- \\ tausch: Die Vielfalt der vorgestellten Projekte zeigt nach wie vor grosse Potentiale \\ zur innovativen Weiterentwicklung der Integrierten Versorgung.
}

Markus Wieser

Der Workshop wurde moderiert von Hansjörg Schlegel und unterstützt von der Firma Novartis Pharma Schweiz AG, vertreten durch Lorenz Borer.

Korrespondenz:

Dr. med. Markus Wieser Medizinischer Leiter

Hawadoc AG

Garnmarkt 1

CH-8400 Winterthur
Die anfängliche provokative These, dass die Managed-Care-Szene nach der abgelehnten MC-Vorlage eingeschlafen und der Begriff sowieso verstaubt sei, weckte alle Teilnehmerinnen und Teilnehmer. Viele neue Projekte werden nun unter dem Titel Integrierte Versorgung subsumiert. Ärzte und Patienten haben sich daran gewöhnt, in gewissen Regionen ist Managed Care schon fast Standard geworden. Daher hat man von MC nicht mehr soviel gehört. Tatsache ist, dass in der Zwischenzeit viele neue Netze entstanden sind. Die Entwicklung neuer Modelle ist ebenfalls im Gang, was die intensiven Diskussionen unter den Netzen und mit Versicherern zeigt. Der ungebremste Anstieg der Versichertenzahlen zeigt eindrücklich, dass das «Hausarztmodell» gefragt ist. Eine Herausforderung, wenn man sich Gedanken zum Hausärztemangel und der Versorgungssicherheit macht und überlegt, wie in integrierten Modellen die Zusammenarbeit intensiviert werden kann.

\section{Managed Care geht in die Zukunft}

Zur Weiterentwicklung von Managed Care wurden die Schwierigkeiten und Engpässe diskutiert. Einigkeit herrschte, dass Managed Care nicht nur ein Einsparmodell, sondern auch ein medizinisches Qualitätsmodell darstellt. Eine fehlende Nationale Strategie und die relativ geringe Entschädigung für den Aufwand schmälern den Erfolg. Es braucht Projekte unter dem Aspekt, dass es mehr Zeit braucht, um zu erklären, warum eine Untersuchung allenfalls unnötig ist, als kurz eine technische Abklärung einzuleiten ... Zur Förderung von Innovationen und neuen Modellen braucht es auch Strukturen unter Einbezug der Hausarztmedizin und der Versicherer. Die gemeinsame Entwicklung fördert die Verbindlichkeit und ermöglicht qualitative Verbesserungen.

\section{Hausarztmangel, Versorgungssicherheit und Praxisnachfolge}

Es ist bekannt, dass es zukünftig schwierig sein wird, gute Hausärztinnen und Hausärzte zu finden. Die Praxen brauchen Nachfolger, aber auch die Netze. Diese brauchen zur Betreuung ihrer Hausarztpatienten direkte Nachfolger, in gewissen Regionen mit flächendeckenden Netzen kann sogar die Frage der Ver- sorgungssicherheit allgemein, aufkommen. Die Kontroverse besteht deshalb darin, dass nicht alle gleichermassen betroffen sind. Ein Projekt zur Patientenversorgung zeigte beispielsweise, dass ein direkter Zugang zur Physiotherapie bei vorgängig ausgewählten Patienten mit klarer Diagnose und Indikation keine Verteuerung der Behandlungswege brachte (zudem klar zufriedene Patienten und Ärzte). Ein weiterer Ansatz steckt in Projekten, die Arbeit der Medizinischen Praxisassistentin weiter aufzuwerten, z.B. im Bereiche der Betreuung von chronisch Kranken wie Diabetesberatung. Die professionelle Organisation von MPA-Qualitätszirkeln mit einheitlichem Standard ist anzustreben. Auch der Praxisnachwuchs junger Ärztinnen und Ärzte mit ihren modernen Bedürfnissen muss früh in ihrer Ausbildung zugegangen werden. Dafür wichtig ist die Vernetzung unter den Grundversorgern mit den Spezialisten sowie der Austausch mit den Spitälern.

Praxisformen und Vernetzung in der Zukunft Es braucht neue Praxisformen, um der heutigen Versorgung und den zukünftigen Kolleginnen und Kollegen gerecht zu werden. Die Tendenz geht weg von der Einzelpraxis und dem unternehmerischen Risiko. Es gibt erfreuliche Projekte, wo Betriebsgesellschaften ihr ökonomisches Wissen und teils auch Geld einfliessen lassen und dadurch die Mediziner wieder Medizin betreiben können. Dies zeigt den erkannten Stellenwerte im Sinne ärzteeigener Strukturen. Die praktische Umsetzung solcher Praxismodelle wurde unterschiedlich beleuchtet. In diesem Zusammenhang wurde auch die weitere elektronische Vernetzung diskutiert. Die Vernetzung mit den Spitälern bezüglich Eintritts-, Austritts- und Medikamentenmanagement sowie der Patientenpfad und dessen Abbildung wurden kontrovers diskutiert. Die Bedürfnisse sind unterschiedlich, aber jeder für sich sucht gute IT-Lösungen bis hin zur OnlinePraxis.

\section{Zukünftige Veränderungen als Risiko und Chance}

Neben den vielen guten Ideen, bestehen auch politische und juristische Gegebenheiten oder Verände- 


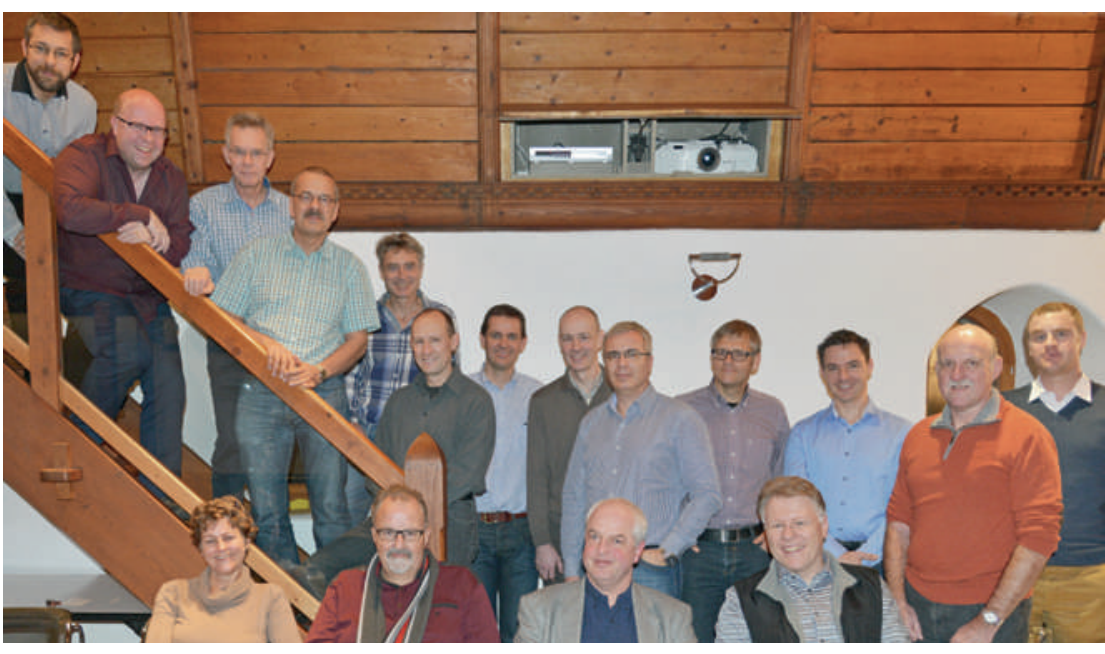

Die Teilnehmer des 16. Managed Care Workshops in Scuol.

rungen. Ab 2014 gelten neue Transparenzregeln für Geldflüsse im Gesundheitssektor. Die Offenlegung von Zuwendungen und Entschädigungen für erbrachte Leistungen machten die Finanzierung durchsichtiger, möglicherweise mit Auswirkung bis auf die Einzelpraxis. In einem Referat und Workshop fand ein Brainstorming statt, wie im bisher klar vom KVG reglementierten Managed Care auch Leistungen im sogenannten «VVG-Bereich» möglich wären. Von den in der Grundversorgung erbrachten Leistungen profitieren ebenfalls Taggeld-, Unfall- und Zusatzversicherer von einer «gemanagten» und koordinierten Führung/Versorgung. Die angedachten Möglichkeiten werden von den Teilnehmern als unterschiedlich realisierbar eingestuft. Einhellig war man der Meinung, dass die Einheitskasse für alle innovativen Projekte der verschiedenen Netze und Garanten negative Auswirkungen hätte. Wir fürchten um kompetente Ansprechpartner, zweifeln an den proklamierten Einsparungen und fürchten eine schlechtere Qualität, starre Regeln und nicht mehr mögliche innovative Projekte für die Bevölkerung.

\section{Medizinische Projekte und Impulsreferat}

Ein Netzwerk lanciert eine Studie zur Therapie bei Harnwegsinfekt, Antibiotika versus nichtsteroidale Antirheumatika mit dem Ziel einer Behandlungsempfehlung. Ein Projekt zeigt am Thema der präoperativen Anämie schön, wie durch gezielte Zusammenarbeit die Operationssicherheit erhöht, die Transfusionsbedürftigkeit gesenkt und dadurch auch Ressourcen eingespart werden können.

Zum Abschluss der Tagung im Sinne einer Horizonterweiterung fand ein Referat der Ethikerin Prof. Dr. med. Dr. phil. Nikola Biller-Andorno vom Institut für Biomedizinische Ethik der Universität Zürich statt. Sie beleuchtete die seit 2012 neu bestehenden amerikanischen «Accountable Care Organizations» (ACO) mit der Frage nach Impulsen für die Schweiz. Diese ACO's wollen das Problem angehen, dass viele Gesundheitsleistungen teuer, falsch eingesetzt und deshalb oft ineffektiv sind. Die Koordination der erbrachten Leistungen im Sinne einer transparenten Behandlungskette soll mehr Qualität bei insgesamt tieferen Kosten bringen. 50-60\% der Einsparungen gehen an die ACO, der Rest an die Versicherung. Prämienrabatte für die Patienten sind kein Anreiz mehr, eine Behandlung kann auch ausserhalb der ACO stattfinden. Eingebunden sind Praxen und Spitäler, einfache Qualitätskriterien sind als lernendes System konzipiert mit dem Ziel, durch Monitoring und Transparenz eine qualitativ hochstehende bedürfnisgerechte ressourcenschonende Versorgung zu erreichen. Unsere Diskussion zeigt, dass wir als Hausärztinnen und Hausärzte nur zu gut verstehen, dass Patienten nicht immer alles wollen, was medizinisch machbar ist. Eine Überversorgung kann sogar zu neuen Problemen/Komplikationen führen. So schliesst sich der Kreis, dass Managed Care durch Koordination eine gute Versorgungsqualität bei geringer steigenden Kosten (als bei unkoordinierter Versorgung) erreicht.

\section{Fazit}

Am Jahresende 2013 konnten an 3 Tagen in Scuol in sehr offener und vertrauter Atmosphäre fernab von Alltag und Praxis brennende Themen der Netzwerkszene diskutiert werden. Das Gezeigte war sehr real, gut fassbar und zeigte die gut schweizerische Vielfalt in der Gestaltungsmöglichkeit im Bereiche der Vernetzung, Zusammenarbeit und Überdenken einer Versorgungssicherheit in unserem Gesundheitswesen. Nach wie vor ist einer der Treiber auch der bevorstehende Hausärztemangel, der neue Innovationen erfordert. Erfreulich auch die Gedanken zur vermehrten Einbindung der MPAs in den Praxen und Etablierung von MPA-Qualitätszirkeln. Die Netze werden auch aktiv in der Nachfolgeplanung von Praxen, investieren in die Erkenntnis, dass junge Kolleginnen und Kollegen vermehrt Teilzeitarbeit wünschen und nicht das Unternehmertum suchen. Wir sind der Meinung, dass die Praxen in ärzteeigener Hand behalten werden und nicht gewinnstrebenden Investoren überlassen werden sollen. 